\title{
Detecting Plagiarism in SolidWorks CAD Courses
}

\section{Dr. Webster R. Johnson, California State University, Chico}

Dr. W.R. Johnson has been a researcher and university professor for the past four decades. He is currently a lecturer at California State University at Chico, lecturing in CAD, thermodynamics, numerical methods, material science and testing, dynamics, and heat transfer. 


\title{
Detecting Plagiarism in SolidWorks CAD Courses
}

\begin{abstract}
The mechanical engineering program at California State University at Chico requires two courses in Computer Aided Design (CAD), Graphics I and Graphics II. The beginning course, usually for first term freshmen, covers the basics of parametric solid modeling, design intent, engineering drawings with proper dimensioning and views, and assemblies. In addition, this first course covers hand sketching and visualization skills. In the second course the students develop and practice more complicated solid modeling challenges including table driven configuration, surface modeling, photo-realistic rendering, motion studies and animation. The two courses are designed to prepare the students for courses in manufacturing, Finite Element Analysis (FEA), Modeling and Simulation, and for the fourth year Capstone project.
\end{abstract}

Both courses utilize SolidWorks, a ubiquitous CAD software in the mechanical engineering discipline. For a single part, a student constructs the solid model saving it in a part (*.SLDPRT) file. The dimensioned representation of the part is then made into an engineering drawing (*.SLDDRW) file.

Until three years ago a persistent problem for the instructors of Graphics I and II was plagiarism: one student would copy another's SLDPRT and SLDDRW files, make a few cosmetic changes to the SLDDRW file, including the "Drawn By" authorship in the title block, and then submit the drawing of the part as his or her own work. While every student would work on the same problem part, the drawings from the class typically show wide variations in the choices of views, placements of the views and the dimensions and, finally, errors. Occasionally when a grader inspects the similar submissions of a large class, two or more drawings appear to differ only by a few superficial relocations of dimensions or views. Holding two such drawings, one atop the other, to a light may suggest but does not prove of plagiarism. An example prepared by the author is shown in Figure 1.

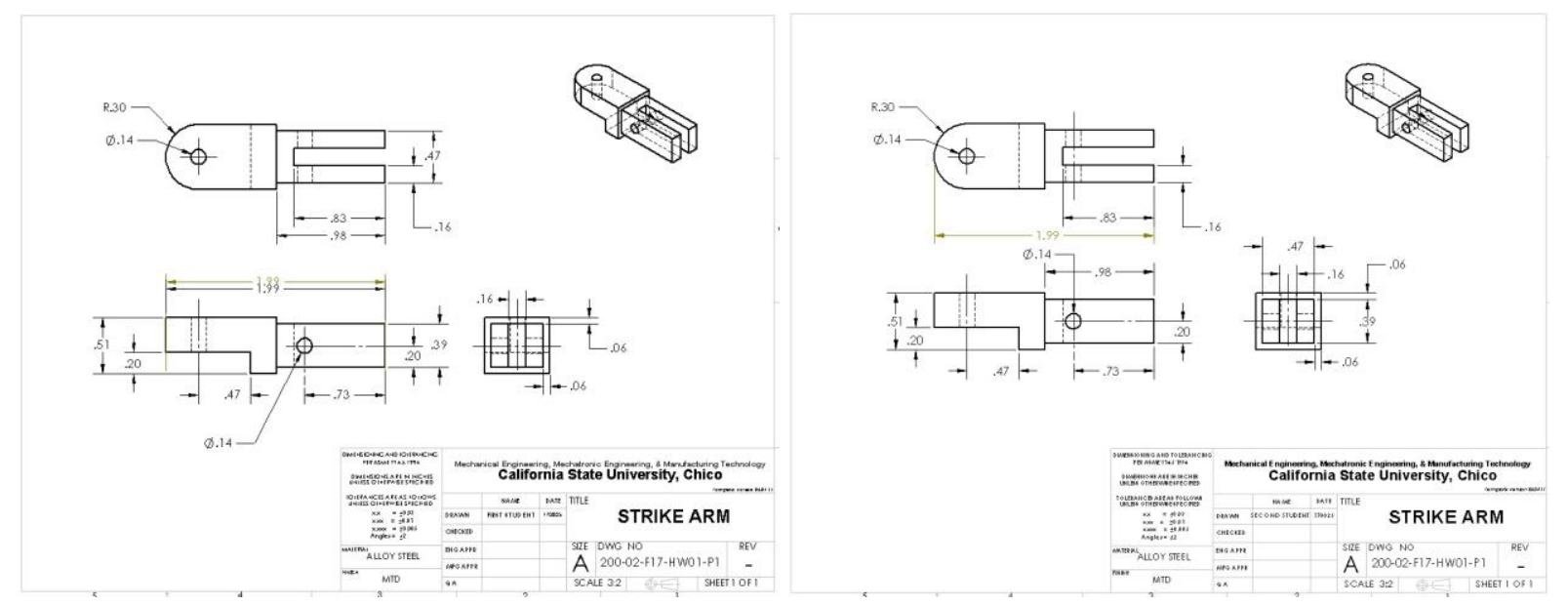

Figure 1: Nearly identical drawings of the same CAD assignment. 
It became obvious to the author that an alternate method of detecting plagiarism was not only desirable but possible. Programming tools available in SolidWorks allowed the quick scanning of the part files instead of the drawing files.

The program developed for detecting plagiarism in SolidWorks files uses method suggested by the experiences of the author and colleagues and from the features in AutoCAD DWG Compare ${ }^{1}$ and Graderworks of Garland Industries ${ }^{2}$.

\section{Method}

In a SolidWorks part file, the construction of an object is a sequence of "features" which are the extrusions, revolves, cut, fillets, holes, etc. which, when geometrically combined, form the part. The features list forms a tree structure and each feature has its own properties: name, description, configuration, the login name of the creator, the date and time of the feature creation, and the date and time of the most recent modification of the feature. The last three properties, Created By (text), Date Created (time), and Last Modified (time) are read-only properties and cannot be altered. Once a feature is created, its Created By and Date Created properties are fixed. The Last Modified property changes when the feature is edited. An example of a feature tree and the feature properties dialog of one feature is shown in Figure 2.

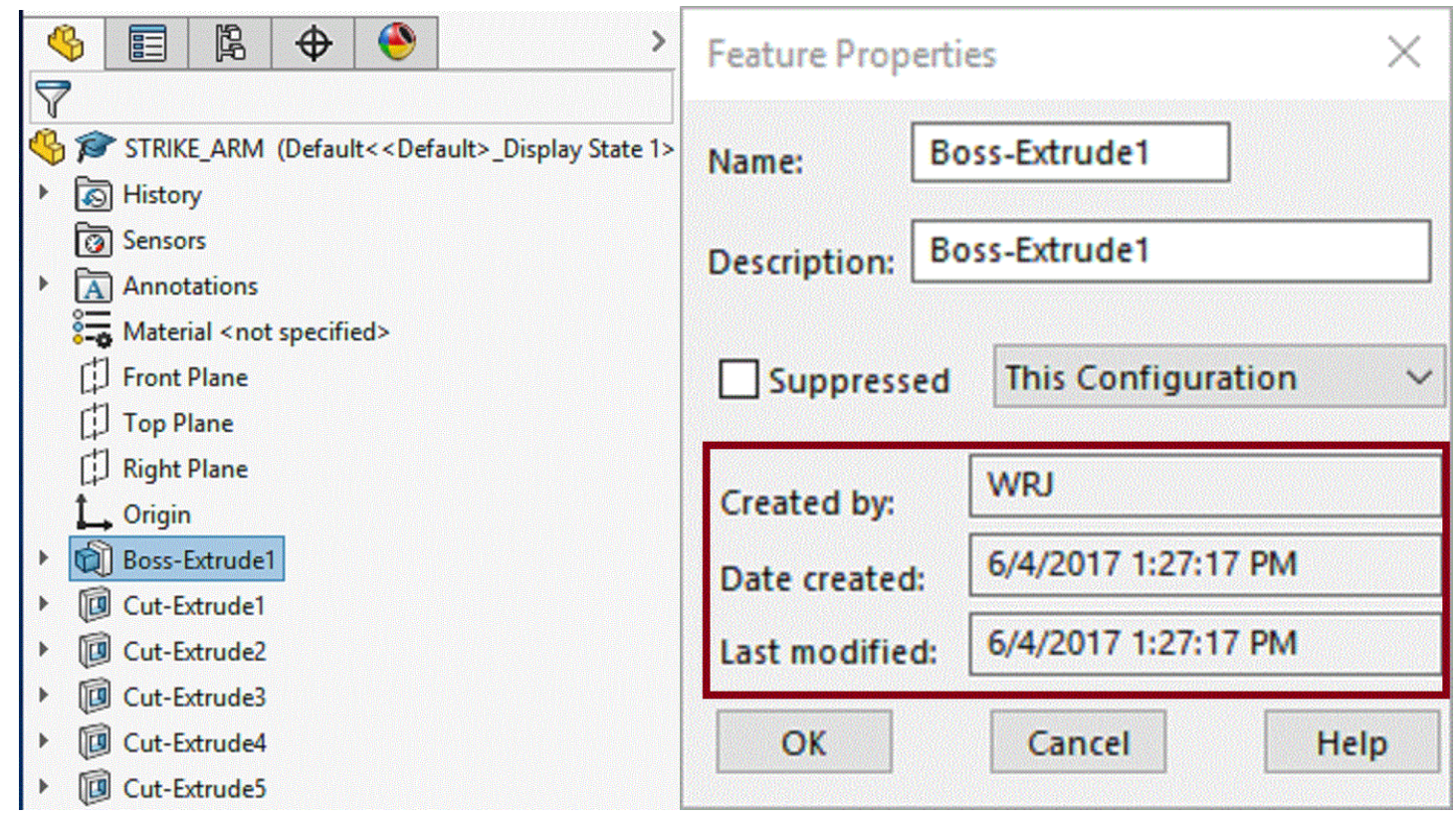

Figure 2: Feature tree (left) and Feature Properties (right)

SolidWorks provides an Application Programming Interface ${ }^{3}$ (API), a set of routines, protocols, and tools for building software applications. A use of the API is to program a new SolidWorks tool, an Add-In, to the main program. The Add-In is programmed to scan the contents of a SolidWorks feature tree of a part file. 
The final Add-In program, named MMEM (after the mnemonic of the mechanical engineering department), examines every feature of a part file. For each feature, the program reads the relevant properties and writes them in an external Microsoft Excel spreadsheet. To ease the inspection of the output the Date Modified is replaced by "Same" if it is identical to the Date Created. Any child member of a feature, such as a 2-D sketch, is also included. A partial listing of the written spreadsheet is shown in Table 1. The relevant entries are the name of the creator of the feature as well as the date of creation and last modification of the feature.

Table 1: Sample of output of the MMEM program

\begin{tabular}{|c|l|l|c|l|}
\hline Name & [Type] & Created By & Date Created & Last Modified \\
\hline$\ldots$ & $\ldots$ & $\ldots$ & $\ldots$ & $\ldots$ \\
\hline Cut-Extrude3 & {$[$ ICE] } & WRJ & $6 / 4 / 2017$ 1:32:13 PM & Same \\
\hline Sketch5 & [ProfileFeature] & WRJ & $6 / 4 / 2017$ 1:30:53 PM & Same \\
\hline Cut-Extrude4 & {$[$ ICE] } & WRJ & $6 / 4 / 2017$ 1:34:06 PM & Same \\
\hline Sketch6 & [ProfileFeature] & WRJ & $6 / 4 / 2017$ 1:32:23 PM & $8 / 28 / 2017$ 12:28:05 PM \\
\hline Cut-Extrude5 & {$[$ ICE] } & WRJ & $6 / 4 / 2017$ 1:35:35 PM & Same \\
\hline Sketch7 & [ProfileFeature] & WRJ & $6 / 4 / 2017$ 1:34:19 PM & Same \\
\hline
\end{tabular}

The user of MMEM may use one of three modes of operation as shown in Figure 3. One may inspect the currently active part file, all of the currently opened part files, or a selection of multiple part files using the familiar Open File dialog box. Selecting any mode starts a new Excel file for recording the list of feature properties. A new worksheet in the Excel file is created for each part file inspected with MMEM. An additional worksheet is prepended with the list of all the file names.

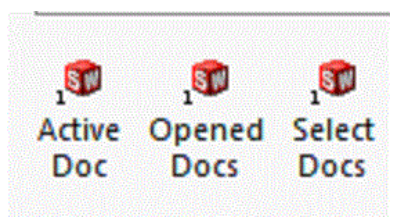

Figure 3: Program options

Students are required to upload their parts files to a specified assignment drop-box on the Learning Management System (LMS) which at CSU, Chico is Blackboard Learn. The instructor then downloads all the part files en masse in a Zip file which he then opens. The LMS prepends information, including the login name, to the user's file name. For example, a file StrikeArmS17. SLDPRT submitted by student with the university login name bandersen 3 for assignment HW 01 will get a new LMS file name:

HW 01_bandersen3_attempt_2017-01-30-00-16-11_Mech200_StrikeArmS17.SLDPRT

With SolidWorks opened and the MMEM add-in enabled, the instructor selects "Select Docs". MMEM opens part file, scans the feature tree, writes a new worksheet in the spreadsheet, closes the part file, and then repeats the sequence with the next part file. Thus a time consuming practice of inspecting each submitted part manually is reduced to a quick scan of all the part features by the program. The instructor, however, must still inspect the results for the 
abnormalities which suggest plagiarism and then decide if the suggestion is positive or is false positive.

\section{Results}

When a large batch of SolidWorks is inspected, the MMEM add-in typically requires 1.8-2.0 seconds for each file. This interval is typical the file of most student projects. Inspecting the spreadsheet results, however, is more time consuming: typically 10-20 seconds is necessary for each file. The desired enhancements discussed below should reduce this latter interval.

The MMEM add-in to SolidWorks was introduced in the fall 2015 semester. Faculty members have used the add-in in five courses. To date cases of plagiarism have been detected exclusively in the freshman and sophomore courses, Graphics I and II, for which the results are listed in Table 2. For the Spring 2016 Graphics I and the Fall 2016 Graphics II, the results are no longer available. The Fall 2018 Graphics I was not offered.

Table 2: Detected Cases of Plagiarism in Graphics I and II during 5 Semesters.

\begin{tabular}{|c|c|c|c|c|c|}
\hline \multirow[t]{2}{*}{ Term } & \multicolumn{2}{|c|}{ Graphics I } & \multicolumn{2}{|c|}{ Graphics II } & \\
\hline & $\begin{array}{l}\text { Student Files } \\
\text { Tested }\end{array}$ & $\begin{array}{l}\text { No. of } \\
\text { Plagiarisms }\end{array}$ & $\begin{array}{l}\text { Student Files } \\
\text { Tested }\end{array}$ & $\begin{array}{l}\text { No. of } \\
\text { Plagiarisms }\end{array}$ & Total \\
\hline Fall 2015 & 1116 & 2 & 810 & 4 & 6 \\
\hline Spring 2016 & - & - & 900 & 3 & 3 \\
\hline Fall 2016 & 870 & 1 & - & - & 1 \\
\hline $\begin{array}{l}\text { Spring } \\
2017\end{array}$ & 852 & 0 & 810 & 0 & 0 \\
\hline Fall 2018 & Not & ffered & 870 & 0 & 0 \\
\hline
\end{tabular}

The number of cases declined during the first three semesters. One may surmise that the students collectively realized that sharing CAD files resulted in penalties. For the last two semesters listed in Table 2, no cases of plagiarism which suggests that the first year students became aware of the instructors' diligence.

The penalty for plagiarism is a failed grade in the course and an incident report which is sent to the office of student judicial affairs. Repeat offenders may be expelled from the university.

The students' responses is anecdotal. No survey has been conducted regarding the antiplagiarism effort.

\section{False Positives}

MMEM may produce a false positive result in at least four situations. A false positive result is one where plagiarism is indicated at first but is not indicated after closer inspection. Fortunately, all are easy to identify. One is due the behavior of SolidWorks, the others due to student behavior. 
Default Part Features: The feature tree of a new SolidWorks part includes more than a dozen default features such as three orthogonal planes, the origin, equations, material, etc. The Created By name reported by MMEM usually shows one or more previous login names instead of that of the student. If the installation of SolidWorks is fresh, the Created By name may even be that of the SolidWorks programmer. The default features, before and including the Origin, must be ignored when the instructor inspects the output for plagiarism.

\begin{tabular}{|c|c|c|c|}
\hline Comments & [CommentsFolder] & fbiordi & 01/23/17 17:48:27 \\
\hline Favorites & [FavoriteFolder] & tmccarthy4 & 01/26/17 14:12:00 \\
\hline History & [HistoryFolder] & tmccarthy 4 & 01/26/17 14:12:00 \\
\hline Selection Sets & [SelectionSetFolder] & tmccarthy4 & 01/26/17 14:12:00 \\
\hline Sensors & [SensorFolder] & tmccarthy 4 & 01/26/17 14:12:00 \\
\hline Design Binder & [DocsFolder] & fbiordi & 01/23/17 17:48:27 \\
\hline Annotations & [DetailCabinet] & fbiordi & 01/23/17 17:48:25 \\
\hline Surface Bodies & [SurfaceBodyFolder] & fbiordi & 01/23/17 17:48:27 \\
\hline Solid Bodies & [SolidBodyFolder] & fbiordi & 01/23/17 17:48:27 \\
\hline Lights, Cameras and Scene & [EnvFolder] & fbiordi & 01/23/17 17:48:27 \\
\hline Equations & [EqnFolder] & fbiordi & 01/23/17 17:48:27 \\
\hline 1060 Alloy & [MaterialFolder] & fbiordi & 01/23/17 17:48:27 \\
\hline Front Plane & [RefPlane] & fbiordi & 01/23/17 17:48:26 \\
\hline Top Plane & [RefPlane] & fbiordi & 01/23/17 17:48:26 \\
\hline Right Plane & [RefPlane] & fbiordi & 01/23/17 17:48:26 \\
\hline Origin & [OriginProfileFeature] & fbiordi & 01/23/17 17:48:27 \\
\hline
\end{tabular}

Sharing a Computer: If two students share the same home computer, MMEM reports the same login name for both. In this case, the Date Created must be inspected. If they are identical then one student simply copied the other. However, if the Date Created time stamps differs, then one student did the assignment after the other and plagiarism is not indicated.

Table 4: MMEM output for two students sharing a computer-same login but different Date Created times

\begin{tabular}{|c|c|c|c|}
\hline Boss-Extrude1 & [Extrusion] & NoLogin & 01/26/17 22:14:38 \\
\hline Sketch1 & [ProfileFeature] & NoLogin & 01/26/17 22:12:16 \\
\hline Boss-Extrude2 & [ICE] & NoLogin & 01/26/17 22:19:53 \\
\hline Sketch2 & [ProfileFeature] & NoLogin & $01 / 26 / 1722: 18: 21$ \\
\hline Cut-Extrude1 & [ICE] & NoLogin & 01/26/17 22:28:40 \\
\hline Sketch3 & [ProfileFeature] & NoLogin & $01 / 26 / 1722: 20: 19$ \\
\hline
\end{tabular}

\begin{tabular}{|c|c|c|c|}
\hline Boss-Extrude1 & [Extrusion] & NoLogin & $01 / 26 / 1723: 25: 10$ \\
\hline Sketch1 & [ProfileFeature] & NoLogin & $01 / 26 / 1723: 20.20$ \\
\hline Boss-Extrude2 & [ICE] & NoLogin & $01 / 26 / 1723: 27.40$ \\
\hline Sketch2 & [ProfileFeature] & NoLogin & $01 / 26 / 1723: 26: 55$ \\
\hline Cut-Extrude1 & [ICE] & NoLogin & $01 / 26 / 1723: 31: 09$ \\
\hline Sketch3 & [ProfileFeature] & NoLogin & $01 / 26 / 1723: 29: 57$ \\
\hline
\end{tabular}

Sharing the Login Credentials: A false positive result appears when two students share the login credentials in a CAD laboratory. This often occurs at the beginning of a term when one student has not updated his or her credentials and is only able to login by using classmate's credentials. 
Fortunately, the two students make the same SolidWorks part but do so at different rates. In this situation, MMEM reports identical Created By names but the Date Created and Date Modified are similar, even overlap, but are not identical. In this case plagiarism is not suspected.

Table 5: Two student sharing login credentials in one lab--same login but different times

\begin{tabular}{|c|c|c|c|}
\hline Boss-Extrude1 & [Extrusion] & rfraga1 & $08 / 25 / 16$ 08:18:43 \\
\hline Sketch1 & [ProfileFeature] & rfraga1 & $08 / 25 / 16$ 08:14:55 \\
\hline Boss-Extrude2 & [ICE] & rfraga1 & $08 / 25 / 16$ 09:02:52 \\
\hline Sketch2 & [ProfileFeature] & rfraga1 & $08 / 25 / 1608: 22: 06$ \\
\hline Fillet1 & [Fillet] & rfraga1 & $08 / 25 / 1609: 03: 08$ \\
\hline Boss-Extrude3 & [ICE] & rfraga1 & $08 / 25 / 1609: 05: 11$ \\
\hline Sketch3 & [ProfileFeature] & rfraga1 & $08 / 25 / 16$ 09:03:51 \\
\hline Cut-Extrude1 & [ICE] & rfraga1 & $08 / 25 / 16$ 09:07:28 \\
\hline Sketch4 & [ProfileFeature] & rfraga1 & $08 / 25 / 16$ 09:06:09 \\
\hline
\end{tabular}

\begin{tabular}{|c|c|c|c|}
\hline Boss-Extrude1 & [Extrusion] & rfraga1 & 08/25/16 08:20:31 \\
\hline Sketch1 & [ProfileFeature] & rfraga1 & $08 / 25 / 16$ 08:15:10 \\
\hline Boss-Extrude2 & [ICE] & rfraga1 & $08 / 25 / 16$ 09:05:30 \\
\hline Sketch2 & [ProfileFeature] & rfraga1 & $08 / 25 / 16$ 08:45:35 \\
\hline Fillet1 & [Fillet] & rfraga1 & $08 / 25 / 16$ 09:06:08 \\
\hline Boss-Extrude3 & [ICE] & rfraga1 & $08 / 25 / 16$ 09:21.15 \\
\hline Sketch3 & [ProfileFeature] & rfraga1 & $08 / 25 / 16$ 09:19,55 \\
\hline Cut-Extrude1 & [ICE] & rfraga1 & $08 / 25 / 1609: 022.58$ \\
\hline Sketch4 & [ProfileFeature] & rfraga1 & $08 / 25 / 16$ 09:22:05 \\
\hline
\end{tabular}

Accidental Case: Only one instance of unintentional plagiarism has been proven. Two students did the same assignment in the same lab. One student did not have a flash drive and saved his work on that of a classmate. Unfortunately he uploaded not his own part file but that of his classmate to the LMS. MMEM reported an unequivocal case of plagiarism: identical Created By, Date Created, and Date Modified feature properties. The instructor inspected the two files on the flash drive to find independent efforts. Plagiarism was not suspected.

\section{Desired Enhancements to MMEM}

The detection of possible cases of plagiarism is accomplished by simple visual inspection of the spreadsheet out of the MMEM program. Using the program to identify cases of outright copying as well as the false positive cases is still a time consuming process. Enhancements to MMEM can shorten the inspection process.

Parse the login name from the LMS file name: The constructed LMS name of the file includes class and section, login name, the university's class numerical id, attempt number, date and time. The login name could be parsed from the file name and compared to the Created By name automatically. However, the match will only occur for laboratory submissions. Homework assignments made on laptops or home desktops will usually not show a positive match. Furthermore, each LMS has a different scheme to construct the file name. The user of MMEM should have a tool for declaring the parsing rules to find the university login from LMS file name.

Other SolidWorks file types: The same MMEM program can also scan drawing (*.SLDDRW) and assembly (*.SLDASM) files. The output, however, is more complicated and does not lend itself to quick visual inspection. The program should be modified to reduce the output to a readable format. 
Compressed files: The program should handle compressed files as well as the standard SolidWorks files. This would eliminated the need of manually decompressing the LMS collection of files. More importantly, it could open and scan assemblies, each of which includes part files and which are often compressed.

Ignoring default features: Most of the default features may be skipped by the MMEM program. Exceptions might include the Equations and Materials features. The user of MMEM should be able to select which features may be ignored.

Comparing Created By names among all the files: Once all the files have downloaded and individual inspected, the MMEM program should compare the Created By names. First, all the Created By names of the non-default features in a given part file should be compared, and, second, the Created By names should be compared among all of the files.

Time intervals: the Date Created and Modified Date should be compared with dates set by instructor. This will quickly reveal a case of self-plagiarism in which a student is trying to reuse work done for a previous class.

Flagging: A more generous use of highlighting the possible inconsistencies in the files should be incorporated.

\section{Conclusion}

The MMEM program has been effective in reducing the incidents of plagiarism in the first and second Graphics courses.

The MMEM program most readily detects such cases when laboratory assignments are involved since each student logs into a workstation with his or her university credentials. However, MMEM cannot always detect plagiarism for homework assignments which are completed on a home computer for which the login differs from the student's university login.

Students may want to the use that "I didn't know that sharing files was forbidden". In the Graphics I and II courses, the instructors have each student read and sign a document in which he or she understands the definition of plagiarism and the academic penalties for violations.

No cases have been determined during the last two semesters. There is no reason to assume that this paucity will continue. Eventually, new students will attempt to copy the work of classmates. Thus, the use of the MMEM program will remain necessary and the enhancements to the program will make inspection less time consuming.

\section{References}

1. Autodesk, https://www.autodesk.com/products/autocad/overview

2. Garland Industries LLC: https://garlandindustriesllc.com/index.php/pages/view/graderworks

3. The SolidWorks API is part of the distribution. The help documents are on-line at http://help.solidworks.com/2017/English/api/sldworksapiprogguide 